\title{
Effect of a Nonionic Surfactant on Biodegradation of Slowly Desorbing PAHs in Contaminated Soils
}

\author{
Marisa Bueno-Montes, ${ }^{\dagger}$ Dirk Springael, ${ }^{\ddagger}$ and José-Julio Ortega-Calvo ${ }^{*,+}$ \\ ${ }^{\dagger}$ Instituto de Recursos Naturales y Agrobiología, C.S.I.C., Apartado 1052, E-41080-Seville, Spain \\ ${ }^{\ddagger}$ Division Soil and Water Management, Catholic University of Leuven, Kasteelpark Arenberg 20, B-3001 Leuven, Belgium. \\ Supporting Information
}

ABSTRACT: The influence of the nonionic surfactant Brij 35 on biodegradation of slowly desorbing polycyclic aromatic hydrocarbons (PAHs) was determined in contaminated soils. We employed a soil originated from a creosote-polluted site, and a manufactured gas plant soil that had been treated by bioremediation. The two soils differed in their total content in five indicator 3-, 4-, and 5-ring PAHs (2923 $\mathrm{mg} \mathrm{kg}^{-1}$ and $183 \mathrm{mg} \mathrm{kg}^{-1}$ in the creosotepolluted and bioremediated soils, respectively) but had a similar content (140 $\mathrm{mg} \mathrm{kg}^{-1}$ vs $156 \mathrm{mg} \mathrm{kg}^{-1}$ ) of slowly desorbing PAHs. The PAHs present in the bioremediated soil were highly recalcitrant. The surfactant at a concentration above its critical micelle concentration enhanced the biodegradation of slowly desorbing PAHs in suspensions of both soils, but it was especially efficient with bioremediated soil, causing a $62 \%$ loss of the total PAH content. An inhibition of biodegradation was observed with the high-molecular-weight PAHs pyrene and benzo[a]pyrene in the untreated soil, possibly due to competition effects with other solubilized PAHs present at relatively high concentrations. We suggest that nonionic surfactants may improve bioremediation performance with soils that have previously undergone extensive bioremediation to enrich for a slowly desorbing profile.

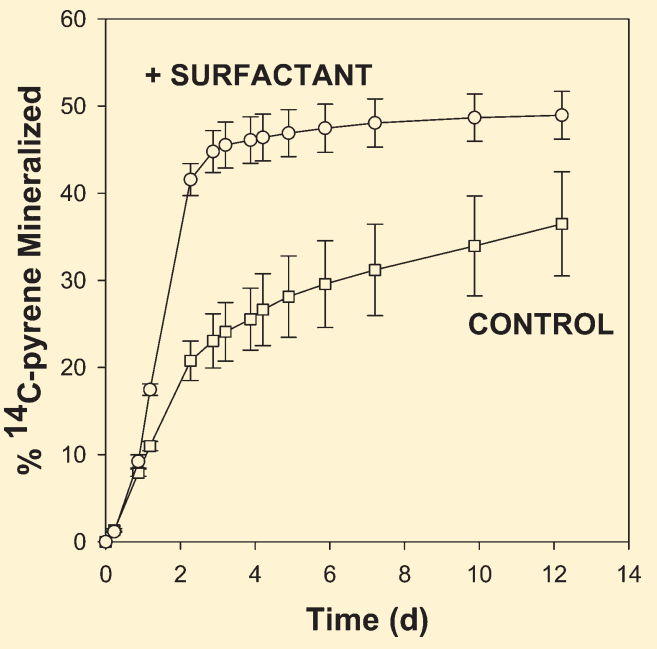

\section{INTRODUCTION}

A major factor limiting the success of bioremediation of polycyclic aromatic hydrocarbons (PAHs) in polluted sites is the low bioavailability of these compounds, due to slow desorption from the solid matrix. ${ }^{1,2}$ The resulting residual concentrations are of strategic value because they can limit future uses of the sites after treatment. Innovative methods are needed, not only to predict the behavior of these recalcitrant pollutant fractions in soils, but also to increase their bioavailability for an enhanced bioremediation performance.

Desorption of PAHs from contaminated soils is well represented by a biphasic pattern, where a first phase of fast desorption (rate constant $>0.1 \mathrm{~h}^{-1}$ ) is followed by a much slower phase, with half-lives that may reach months or years. ${ }^{3}$ Slow desorption can be prominent if soils are enriched in compartments such as semisolid, NAPL-like creosote materials, which limit biodegradation due to a restricted surface area and a slow diffusion inside the organic phase, ${ }^{4,5}$ or black carbon-type materials, with a strong affinity for sorption of PAHs. ${ }^{6}$ The desorption pattern of PAHs can be seriously affected when the pollutants remain in soils for long time periods, due to aging phenomena that increase the pool of slowly desorbing chemicals, with the subsequent reduction in biodegradation rates. ${ }^{7}$ Based on an extensive literature review on desorption, solubilization, and/or biodegradation performance of various surfactants, ${ }^{8-13}$ it is conceivable that nonionic surfactants can increase biodegradation rates of slowly desorbing PAHs in soils. In general, the nonionic surfactant group is the most frequently used in biodegradation studies, mainly due to the absence of electrical charge in the molecule of surfactant, what minimizes eventual toxic effects. Furthermore, in comparison with cationic or anionic surfactants, the nonionic group also shows in general a lower critical micelle concentration (CMC). Despite its potential in bioremediation, the capacity of nonionic surfactants to enhance biodegradation rates of slowly desorbing PAHs remains relatively unexplored. Whereas the promoting effect of nonionic surfactants on solubilization of PAHs from soils is a well-known phenomenon, ${ }^{12,13}$ studies reporting precise measurements of biodegradation rates for slowly desorbing PAHs in the presence of surfactants are very scarce. The only available assessment was recently provided with the nonionic surfactant Brij 30 for contaminated soil from a manufactured gas plant site (MGP) that had first been treated in an aerobic bioreactor. ${ }^{14}$ By using a single-step, slurry incubation during 18

Received: October 22, 2010

Accepted: February 21, 2011

Revised: February 14, 2011

Published: March 04, 2011 
days, the study evidenced surfactant-enhanced desorption and biodegradation of residual PAHs in the bioremediated soil, whereas the surfactant had no effect on biodegradation in the untreated soil. However, the magnitude of the slowly desorbing fraction of PAHs present in the soils was not determined.

We employed for this research two contaminated soils; one of them originated directly from a creosote-polluted site, whereas the other was a MGP soil that had been previously treated extensively by bioremediation. The soils differed in their total content in PAH but had a similar content of slowly desorbing $\mathrm{PAH}$, as determined by Tenax extraction. According to the literature ${ }^{8-13}$ and our own experience, ${ }^{15,16}$ we selected Brij 35 , an nonionic alkylpoly (ethylene glycol) ether surfactant, for our study. We determined the effect of the surfactant at a concentration above its CMC on biodegradation of slowly desorbing fractions of PAHs in these soils through a dual radiorespirometry/residue analysis method, what allows precise estimations of compound bioaccessibility. ${ }^{16,17}$

\section{MATERIALS AND METHODS}

Chemicals. $\left[9{ }^{14} \mathrm{C}\right]$-phenanthrene $\left(13.1 \mathrm{mCi} \mathrm{mmol}^{-1}\right)$, $[1,2,3,4,4 \mathrm{a}, 9 \mathrm{a}-14 \mathrm{C}]$-anthracene $\left(20.6 \mathrm{mCi} \mathrm{mmol}^{-1}\right),\left[3^{-14} \mathrm{C}\right]$ fluoranthene $\left(45.0 \mathrm{mCi} \mathrm{mmol}^{-1}\right)$ and $\left[4,5,9,10-{ }^{14} \mathrm{C}\right]$-pyrene $\left(58.7 \mathrm{mCi} \mathrm{mmol}^{-1}\right)$, all of them with a radiochemical purity $>98 \%$, were provided by Sigma Chemical Co., St. Louis, MO. The nonionic alkyl poly(ethylene glycol) ether surfactant Brij 35 $\left[\mathrm{C}_{12} \mathrm{H}_{25} \mathrm{O}\left(\mathrm{CH}_{2} \mathrm{CH}_{2} \mathrm{O}\right)_{23} \mathrm{H}\right]$ was purchased from Sigma-Aldrich (Steinheim, Germany). Tenax $(60-80$ mesh, 177-250 $\mu \mathrm{m})$ was supplied by Chrompack.

Soils. Two soils were used in this study: a creosote-polluted soil and a bioremediated soil. The former was a clay soil, classified as a calcaric fluvisol, provided by EMGRISA (Madrid, Spain) from an abandoned wood-treating facility in Andújar (Jaen, Spain), with a record of pollution by creosote going back 100 years. This soil was obtained from a point with heavy pollution (4501 $\mathrm{mg} \mathrm{kg}^{-1}$ total PAH, sum of $16 \mathrm{EPA} \mathrm{PAH),} \mathrm{and} \mathrm{had} \mathrm{3 \%}$ organic matter and $60 \%$ clay. A detailed description of this soil is given elsewhere. ${ }^{15}$ The bioremediated soil originated from a Danish manufactured gas plant (MGP) site. It was obtained from a remediation company (Soilrem, Kalundborg, Denmark) that had treated the soil in biopiles during several years to bring the total PAH concentration down to $275 \mathrm{mg} \mathrm{kg}^{-1}$ (16 EPA PAH). These residual PAHs exhibited a high resistance to dissipation, according to further bioremediation efforts performed by the company that included organic amendments and composting. ${ }^{2}$ The soil had $3.6 \%$ organic matter, $28.8 \%$ clay and a $\mathrm{pH}$ of 7.96 . These soils were air-dried, sieved ( $2 \mathrm{~mm}$ mesh $)$ and kept in glass containers in the dark at $4{ }^{\circ} \mathrm{C}$ until use.

Desorption. The procedure for desorption experiments is similar to the one previously described. ${ }^{3}$ Briefly, $0.5 \mathrm{~g}$ dry soil, $35 \mathrm{~mL}$ milli-Q water, $0.2 \mathrm{~mL}$ formaldehyde (40\%), and $0.7 \mathrm{~g}$ Tenax TA beads were placed in $50 \mathrm{~mL}$ stainless steel centrifuge tubes equipped with a stainless steel sealing (Heraeus-Sorvall, Madrid) and kept at $23 \pm 2{ }^{\circ} \mathrm{C}$ and $120 \mathrm{rpm}$ on an rotary shaker. After certain time intervals, the tubes were centrifuged for $10 \mathrm{~min}$ at $17212 \mathrm{~g}$. The floating Tenax beads were completely recovered with a spatula without disturbing the soil pellet. Then, the same amount of fresh Tenax beads was added to the tubes, the soil pellet was resuspended, and the cycle was repeated.

The Tenax recovered from the tubes was extracted with $100 \mathrm{~mL}$ of acetone/hexane $(1: 1)$ in $250 \mathrm{~mL}$ screw-capped
Erlenmeyer flasks that were kept for $5 \mathrm{~h}$ on a rotary shaker operating at $120 \mathrm{rpm}$. The extract was evaporated to near dryness, redissolved in acetonitrile, and filtered. $\mathrm{PAH}$ analysis was performed by HPLC as described below. The total mass of PAH desorbed plus the amount still present in the soil at the end of the experiments was for phenanthrene and pyrene higher than $98 \%$ in the creosote-polluted soil. Recoveries were in all other cases higher than $80 \%$, with the exception of fluoranthene $(69.3 \%)$ and benzo[a]pyrene (76\%) in the creosote-polluted soil and anthracene $(58.1 \%)$ in the bioremediated soil.

Desorption data could be empirically described by the following first-order, two-compartment kinetic model: ${ }^{1}$

$$
S_{t} / S_{\mathrm{o}}=F_{\text {fast }} \exp \left(-k_{\text {fast }} t\right)+F_{\text {slow }} \exp \left(-k_{\text {slow }} t\right)
$$

where $S_{t}$ and $S_{\mathrm{o}}(\mathrm{g})$ are the soil-sorbed amounts of PAHs at time $t$ (h) and at the start of the experiment, respectively, $F_{\text {fast }}$ and $F_{\text {slow }}$ are the fast- and slow-desorbing fractions, and $k_{\text {fast }}$ and $k_{\text {slow }}$ $\left(\mathrm{h}^{-1}\right)$ are the rate constants of fast and slow desorption. The values of $F_{\text {fast }}, F_{\text {slow, }}, k_{\text {fast }}$ and $k_{\text {slow }}$ were obtained by minimizing the cumulative squared residuals between experimental and calculated values of $\ln \left(S_{t} / S_{\mathrm{o}}\right)$. The software used for the minimization was Microsoft Excell 2003 (Solver option).

Surfactant Sorption onto Soil and Solubilization of Soil PAHs by Surfactant Micellar Solutions. Sorption of the surfactant onto the soil was assessed by measuring the increase in critical micelle concentration (CMC) in soil suspensions, as compared to aqueous solution. The CMC was calculated as the lowest surfactant concentration not leading to a significant decrease in surface tension of an inorganic salts solution ( $\mathrm{pH}$ 5.7, described elsewhere ${ }^{17}$ ). The CMC in soil suspensions was determined with supernatants obtained after centrifuging $(17212 \mathrm{~g}, 15 \mathrm{~min})$ suspensions of creosote-polluted soil containing $1 \mathrm{~g} / 70 \mathrm{~mL}$ inorganic salts solution, that were equilibrated with different surfactant concentrations during $24 \mathrm{~h}$ in a rotary shaker. Surface tension was determined at $25{ }^{\circ} \mathrm{C}$ with a TD1 Lauda ring tensiometer and plotted as $\mathrm{dyn} / \mathrm{cm}$ against the logarithm of surfactant concentration in $\mathrm{mg} / \mathrm{L}$.

The solubilization of PAHs in the presence of aqueous-phase Brij 35 was determined in $50 \mathrm{~mL}$ steel centrifuge tubes having a suspension that contained $0.5 \mathrm{~g}$ dry soil, $35 \mathrm{~mL}$ inorganic salts solution, $0.2 \mathrm{~mL}$ formaldehyde (40\%), and $5 \mathrm{~mL}$ of a Brij 35 solution to give a final surfactant concentration of $20 \mathrm{~g} / \mathrm{L}$. The suspensions were maintained under the same conditions as Tenax desorption experiments. After certain time intervals, the tubes were centrifuged for $10 \mathrm{~min}$ at $17212 \mathrm{~g}$, and an aliquot of the supernatant was analyzed for PAHs. The remaining supernatant was carefully decanted without disturbing the soil pellet, fresh surfactant solution was added to the tube, and the washing cycle was repeated. The complete procedure involved at least five washing steps performed during $168 \mathrm{~h}$.

Biodegradation. The microbial accessibility of native PAHs was assessed through a dual ${ }^{14} \mathrm{C} /$ residue analysis method. ${ }^{17}$ For this aim, $1 \mathrm{~g}$ of dry soil was placed in $250 \mathrm{~mL}$ Erlenmeyer flasks, and mixed with $1 \mathrm{~mL}$ of a sterile, inorganic salts solution, that contained between 80000 and $100000 \mathrm{dpm}$ of the corresponding ${ }^{14} \mathrm{C}$-labeled PAH (phenanthrene, anthracene, fluoranthene, and pyrene - benzo[a]pyrene was not expected to be mineralized under the experimental conditions used). According to the specific activity of the stocks, the label added corresponded to a negligible fraction of the native PAH-load in the soils. The ${ }^{14} \mathrm{C}$ labeled PAH were added completely dissolved in the aqueous phase, a procedure that guaranteed the homogenization of the 
Table 1. Desorption Kinetics, As Obtained with Tenax, And Solubilization by an Anionic Surfactant $\left(\mathrm{Brij}^{35}, 20 \mathrm{~g} \mathrm{L^{-1 }}\right) \mathrm{of}$ Polycyclic Aromatic Hydrocarbons (PAHs) from Polluted Soils

\begin{tabular}{|c|c|c|c|c|c|c|c|c|c|c|}
\hline \multirow[b]{2}{*}{ PAH } & \multicolumn{5}{|c|}{ creosote-polluted soil (Andújar site) } & \multicolumn{5}{|c|}{ bioremediated soil from manufactured gas plant site (Soilrem) } \\
\hline & $\begin{array}{l}\text { PAH concentration } \\
\quad\left(\mathrm{mg} \mathrm{kg}^{-1}\right)\end{array}$ & $\begin{array}{l}k_{\text {fast }}^{a} \\
\left(h^{-1}\right)\end{array}$ & $\begin{array}{c}k_{\text {slow }}{ }^{a} \\
\left(10^{-3} \mathrm{~h}^{-1}\right)\end{array}$ & $\begin{array}{l}F_{\text {fast }}^{a} \\
(\%)\end{array}$ & $\begin{array}{c}F_{\mathrm{Brij35}}{ }^{b} \\
(\%)\end{array}$ & $\begin{array}{l}\text { PAH concentration } \\
\quad\left(\mathrm{m} \mathrm{kg}^{-1}\right)\end{array}$ & $\begin{array}{l}k_{\mathrm{fast}}^{a} \\
\left(\mathrm{~h}^{-1}\right)\end{array}$ & $\begin{array}{c}k_{\text {slow }}{ }^{a} \\
\left(10^{-4} \mathrm{~h}^{-1}\right)\end{array}$ & $\begin{array}{l}F_{\text {fast }}{ }^{a} \\
(\%)\end{array}$ & $\begin{array}{c}F_{\mathrm{Brij} 35}^{b} \\
(\%)\end{array}$ \\
\hline phenanthrene & $1329.5 \pm 52.7$ & $0.38 \pm 0.00$ & $2.70 \pm 0.03$ & $96.1 \pm 0.1$ & $102.0 \pm 2.4$ & $42.3 \pm 5.6$ & $0.16 \pm 0.00$ & $1.6 \pm 0.1$ & $12.0 \pm 1.6$ & $62.3 \pm 0.3$ \\
\hline anthracene & $286.3 \pm 9.1$ & $0.26 \pm 0.04$ & $2.69 \pm 1.70$ & $94.6 \pm 0.6$ & $107.0 \pm 1.2$ & $15.4 \pm 1.5$ & $0.13 \pm 0.02$ & $1.2 \pm 0.1$ & $9.6 \pm 1.8$ & $32.0 \pm 1.4$ \\
\hline fluoranthene & $881.5 \pm 3.4$ & $0.30 \pm 0.02$ & $2.40 \pm 0.58$ & $95.6 \pm 1.1$ & $93.2 \pm 2.6$ & $54.8 \pm 4.3$ & $0.14 \pm 0.00$ & $1.9 \pm 0.1$ & $18.3 \pm 4.0$ & $35.2 \pm 10.6$ \\
\hline pyrene & $395.2 \pm 15.0$ & $0.26 \pm 0.03$ & $2.20 \pm 0.50$ & $92.3 \pm 1.1$ & $99.3 \pm 1.0$ & $48.3 \pm 6.7$ & $0.15 \pm 0.01$ & $1.5 \pm 0.0$ & $15.1 \pm 1.9$ & $32.2 \pm 5.2$ \\
\hline benzo[a]pyrene & $30.7 \pm 4.4$ & $0.11 \pm 0.03$ & $1.80 \pm 0.20$ & $86.7 \pm 0.9$ & $\mathrm{ND}^{c}$ & $22.4 \pm 0.1$ & $0.06 \pm 0.00$ & $1.3 \pm 0.1$ & $13.7 \pm 1.4$ & $23.8 \pm 2.8$ \\
\hline
\end{tabular}

labeled compounds within the soil. Then, more inorganic salts solution was added to complete a final volume of $70 \mathrm{~mL}$. The solution contained, when appropriate, Brij 35 to give a final concentration of $20 \mathrm{~g} \mathrm{~L}^{-1}$. The flasks were then closed with Teflon-lined stoppers, from which a $2 \mathrm{~mL}$ vial containing $1 \mathrm{~mL}$ of $0.5 \mathrm{M} \mathrm{NaOH}$ was suspended to trap ${ }^{14} \mathrm{CO}_{2}$. The flasks were incubated at $23 \pm 2{ }^{\circ} \mathrm{C}$ on an orbital shaker operating at $100 \mathrm{rpm}$. Mineralization of ${ }^{14} \mathrm{C}-\mathrm{PAH}$ in these biostimulated soil suspensions was then measured as described elsewhere. ${ }^{17}$ Mineralization rates were calculated and compared as the slope of the regression lines drawn with the points belonging to the phase of maximum mineralization. The final extents of mineralization were not considered in our bioaccessibility assessment. Instead, residual contents of native PAH were determined in separate, duplicate flasks which were incubated under the same conditions but contained no ${ }^{14} \mathrm{C}$-labeled compound. At the end of the assay (mineralization rate $<0.1 \%$ per day ${ }^{17}$ ), the flasks were sacrificed and kept frozen at $-80{ }^{\circ} \mathrm{C}$ until analysis for PAH content by HPLC, both in the soil and in the aqueous phase.

Mycobacterium gilvum VM552 was used in some experiments to inoculate soil suspensions. The bacterium is able to degrade simultaneously in soil, through growth-linked or cometabolic reactions, phenanthrene, anthracene, fluoranthene, pyrene, and benzo[a]pyrene, and it is not able to degrade Brij 35. The strain was cultured with phenanthrene as the sole source of carbon and energy and prepared for biodegradation experiments as previously described. ${ }^{15}$ Each flask received approximately $10^{7}$ cells $\mathrm{g}^{-1}$ soil. Inoculated suspensions were prepared as above with sterile soil that had been autoclaved three times. Preliminary experiments showed that this sterilization procedure was efficient to prevent any biodegradation activity during the experimental period in the absence of inoculation. The suspensions were thoroughly mixed in a rotary shaker at $120 \mathrm{rpm}$ for $72 \mathrm{~h}$ before inoculation.

Analysis of Native PAH. Soil samples were defrosted, dried at $40{ }^{\circ} \mathrm{C}$, ground with a mortar and pestle, and extracted in a Soxhlet with $100 \mathrm{~mL}$ dichloromethane for $8 \mathrm{~h}$. Acetone was used instead of dichloromethane to extract the soil samples from surfactant treatments. Controls run to test the recoveries of PAHs from soils exposed to the surfactant were in all cases higher than $98 \%$. Once obtained the extract, the organic solvent was evaporated in vacuum to almost dryness, and the residue was dissolved in $5 \mathrm{~mL}$ dichloromethane and cleaned by passing through a Sep-Pak Florisil cartridges. The purified extracts were evaporated with $\mathrm{N}_{2}$ to near dryness and the residue was redissolved in acetonitrile. Then, the samples were filtered through $0.45 \mu \mathrm{m}$ filter nylon.
PAH analysis was carried out using a Waters HPLC system (2690 separations module and Water 996 photodiode array detector, Water PAH column $\mathrm{C}_{18}, 5 \mu \mathrm{m}$ particle size and $4.6 \times 250 \mathrm{~mm}$,

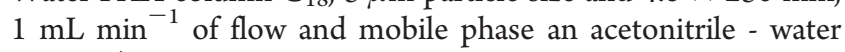
gradient). The column was installed in a thermostatic oven at $30{ }^{\circ} \mathrm{C}$. To complete the mass balances in biodegradation experiments, the PAHs present in the aqueous phase from soil suspensions was also analyzed by direct injection into the HPLC.

\section{RESULTS}

Spontaneous Desorption. The desorption kinetics of phenanthrene, anthracene, fluoranthene, pyrene and benzo[a]pyrene present in creosote-polluted and MGP soils were determined, under abiotic conditions, by Tenax extraction. Figure S1 (Supporting Information (SI)) shows the results for representative desorption experiments, and indicates that the model (eq 1) allows a good prediction of spontaneous desorption. The results with the five compounds and two soils were successfully fitted, and the parameter values are shown in Table 1. The kinetic analysis of desorption showed, with all compounds in a given soil, a similar distribution between the fast- and slowdesorbing fractions. The fast-desorbing fraction accounted for approximately $90 \%$ of the total amount of soil $\mathrm{PAH}$ in the creosote-polluted soil, whereas this fraction was significantly reduced (9.6-18.3\%) in the MGP soil. The MGP soil was therefore enriched in $\mathrm{PAH}$ exhibiting slow desorption, what is in agreement with previous observations on PAH-polluted soils and sediments which have undergone extensive bioremediation. ${ }^{1-3}$ The sum of absolute concentrations of the five $\mathrm{PAH}$ fractions exhibiting slow desorption was comparable in both soils (140.4 mg kg-1 and $156.2 \mathrm{mg} \mathrm{kg}^{-1}$ for creosotepolluted and MGP soils, respectively). However, the value for $k_{\text {slow }}$ of individual PAHs was 10 -fold higher in the creosotepolluted soil than in MGP soil, resulting half-lives for slow desorption of the order of 14 and 200 days, respectively.

Solubilization of Slowly Desorbing PAH by Surfactant. Our working hypothesis was based on possible effects on biodegradation of slowly desorbing PAHs caused by an enhanced solubilization in the presence of aqueous-phase surfactant micelles. Therefore, sufficient Brij 35 was needed in biodegradation experiments that guaranteed the presence of surfactant in solution during incubations, accounting for possible losses of aqueous-phase surfactant due to sorption onto soil and, especially at later stages, to biodegradation of the surfactant itself. The CMC values calculated for aqueous solutions and creosote-polluted soil 


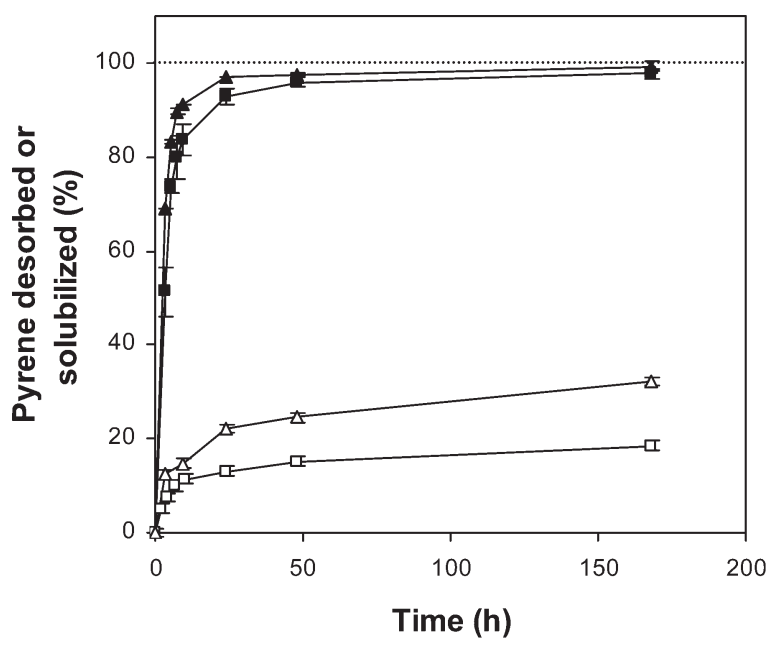

Figure 1. Effect of a nonionic surfactant (Brij 35, triangles) on solubilization of pyrene from a creosote-polluted soil (dark symbols) and a bioremediated soil from a manufactured gas plant site (white symbols), as compared with the spontaneous desorption of the chemical determined with Tenax extraction (squares).

suspensions were, respectively, 77 and $817.6 \mathrm{mg} \mathrm{L}^{-1}$, concentrations at which a minimal surface tension of $57 \pm 1 \mathrm{dyn} \mathrm{cm}^{-1}$ was achieved (SI Figure S2). The difference between these two values indicates that most of the surfactant was sorbed at that soil suspension CMC value (90.5\%, giving a solid-phase surfactant concentration of $\left.53 \mathrm{mg} \mathrm{g}^{-1}\right)$, and evidence the maximum sorption capacity of soil for this nonionic surfactant under the experimental conditions used. ${ }^{10}$ According to these estimations, to the similar content in organic matter in both soils and to preliminary biodegradation experiences, we chose $20 \mathrm{~g} \mathrm{~L}^{-1}$ as the surfactant concentration for all experiments, which was approximately 25 times the CMC in soil suspensions. The surfactant at this concentration mobilized a significant fraction of the slowly desorbing PAHs present in both soils. Figure 1 shows results for the time course of solubilization of pyrene in the presence of the surfactant, as compared to Tenax-driven desorption. Solubilization was especially significant with MGP soil. Taking into account that $85 \%$ of pyrene was present in this soil as slowly desorbing compound with $k_{\text {slow }}=1.5 \times 10^{-4} \mathrm{~h}^{-1}$, a very low amount of this PAH (2.1\% of the total) was expected to be released from this slow fraction after $168 \mathrm{~h}$. However, the fraction extracted by the surfactant at this time was $32.2 \%$, doubling the fast-desorption fraction (15.1\%). A similar effect was observed in MGP soil with the other PAHs studied (Table 1). With the creosote-polluted soil, solubilization of the slowly desorbing $\mathrm{PAH}$ by the surfactant was evidenced for phenanthrene, anthracene, and pyrene by the complete recovery of the PAH mass initially present in the soil (Table 1). Experimental evidence for an enhanced solubilization of slowly desorbing benzo[a]pyrene by the surfactant could not be obtained through this approach with this soil due to analytical interferences caused by the surfactant and the other PAHs.

Biodegradation. Biostimulation experiments with creosotepolluted soil showed the sequential respiration of the $\mathrm{PAH}$, that is, a significant fraction of ${ }^{14} \mathrm{C}$-phenanthrene carbon was recovered as ${ }^{14} \mathrm{CO}_{2}$ before the onset in the mineralization of pyrene (Figures $2 \mathrm{~A}$ and $\mathrm{B}$ ). Mineralization of anthracene and fluoranthene behaved in a similar way as pyrene, with acclimation phases of 5 and 10 days, respectively (data not shown). This biodegradation pattern is often observed during bioremediation of unweathered, PAH-polluted soils. ${ }^{18}$ The addition of Brij 35 did not induce a significant change in this sequential pattern. However, the maximum rate of mineralization was significantly enhanced for the four compounds tested (Table 2). Furthermore, the measurement of residual PAH concentrations evidenced the promoting effect of the surfactant also on the biodegradation of the slowly desorbing fractions of phenanthrene, anthracene and fluoranthene, whose concentrations fell below predictions from $F_{\text {fast }}$ (Table 2). However, the surfactant inhibited the biodegradation of slowly desorbing pyrene, and prevented completely the dissipation of benzo[a]pyrene. Possibly for this reason, $77 \%$ of residual benzo[a]pyrene was detected in the aqueous phase at the end of the experimental period, whereas the aqueous-phase concentrations of the other four $\mathrm{PAH}$ remained below the detection limit. The surface tension of the aqueous phase $\left(59 \pm 1 \mathrm{dyn} \mathrm{cm}^{-1}\right)$ confirmed the presence of the surfactant at this stage. This value corresponds to a surfactant aqueous-phase concentration below CMC (SI Figure S2), and it could be explained by the biodegradation of the surfactant by autochthonous soil microorganisms.

The effect of the surfactant on biodegradation of PAHs in creosote-polluted soil was also tested in suspensions inoculated with phenanthrene-grown M. gilvum VM552 cells (Figure 2C,D and Table 2). The activity of the autochthonous microorganisms was prevented in this experiment by autoclaving. The bacterium mineralized simultaneously and without a lag phase ${ }^{14} \mathrm{C}$-labeled phenanthrene, pyrene and-not shown in Figure 2-fluoranthene. The maximum rates of mineralization of these PAHs were enhanced significantly in the presence of Brij 35 (Table 2). In the control assay without surfactant, the analysis of residual concentration of phenanthrene remaining at the end of the mineralization plateau evidenced a dissipation of the compound higher than predicted by $F_{\text {fast }}$ (Table 2), what indicates that part of the slowly desorbing chemical was also degraded. On the contrary, $F_{\text {fast }}$ overpredicted the extent of biodegradation of the other four PAH (anthracene, fluoranthene, pyrene and benzo[a]pyrene), possibly because spontaneous desorption exceeded, under these conditions, the catabolic potential of microorganisms. In the presence of surfactant, biodegradation brought residual concentrations of phenanthrene and pyrene to undetectable levels, and it yielded lower residuals for anthracene and fluoranthene, as compared with the control. As a result of this enhancement, the total concentration of the five PAH after biodegradation $\left(51.2 \mathrm{mg} \mathrm{kg}^{-1}\right)$ was lower than with surfactantcontaining biostimulated soil suspensions (76.4 $\mathrm{mg} \mathrm{kg}^{-1}$ ), and the PAH levels predicted by $F_{\text {fast }}\left(140.4 \mathrm{mg} \mathrm{kg}^{-1}\right)$. This is remarkable in spite of the short experimental period considered (12 days). The dissipation of benzo[a]pyrene was, however, not promoted by Brij 35. The surface tension of the aqueous phase was, at the end of the experiment, $56.7 \pm 0.3 \mathrm{dyn} \mathrm{cm}^{-1}$, confirming the presence of surfactant micelles in solution (SI Figure S2). This result is consistent with the inability of $M$. gilvum VM552 to utilize Brij 35 as a carbon or energy source.

Biostimulation results of MGP soil showed without surfactant the simultaneous mineralization, without a significant lag phase, of ${ }^{14} \mathrm{C}$-labeled phenanthrene, anthracene, fluoranthene and pyrene (see Figure 2E and SI Figure S3). This was expected because of the history of this soil. However, measurements of final concentrations of native $\mathrm{PAH}$ in these conditions revealed negligible biodegradation. Only fluoranthene and pyrene 

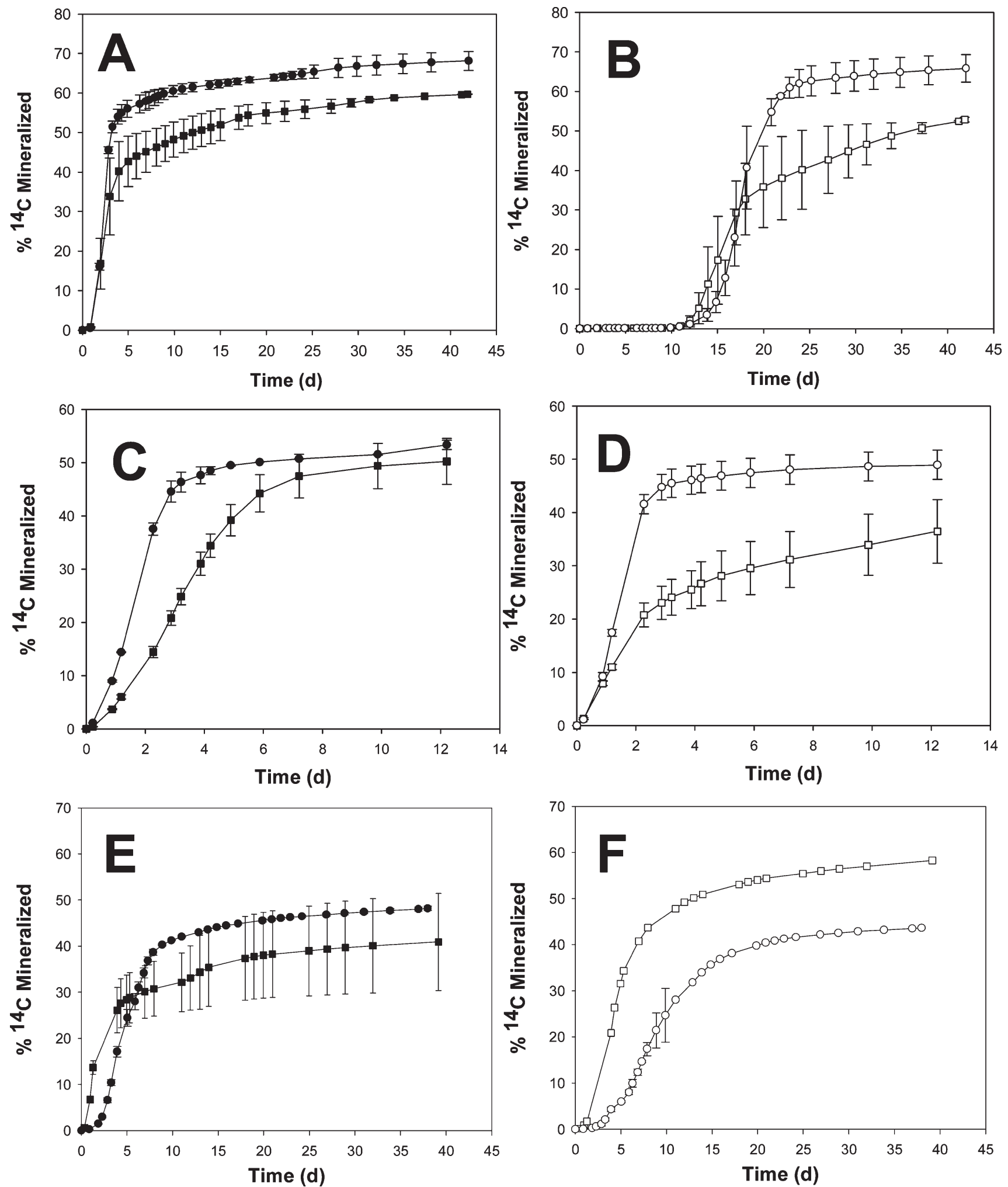

Figure 2. Mineralization of phenanthrene (dark symbols in A, C, and E) and pyrene (white symbols in B, D, and F) in the absence (squares) and presence (circles) of a nonionic surfactant (Brij 35). A and B, biostimulated creosote-polluted soil suspensions; C and D, inoculated creosote-polluted soil suspensions; E and F, suspensions with bioremediated soil from a manufactured gas plant site.

concentrations decreased slightly at the end of the experimental period of 38 days (Table 3). With surfactant, a significant reduction was observed in the residual concentrations of the five $\mathrm{PAH}$, what resulted in a $62 \%$ loss of the total PAH content. The resulting levels fell in all cases below threshold predictions from $F_{\text {fast }}$. This effect was, however, not accompanied by increases in maximum rates of mineralization of the ${ }^{14} \mathrm{C}$-labeled PAHs. Instead, the surfactant caused a short lag phase before the onset 
Table 2. Effect of an Anionic Surfactant (Brij 35, $20 \mathrm{~g} \mathrm{~L}^{-1}$ ) on Biodegradation of Polycyclic Aromatic Hydrocarbons (PAHs) in Suspensions of a Creosote-Polluted soil (Andújar site)

\begin{tabular}{|c|c|c|c|c|c|c|c|c|c|c|}
\hline \multirow[b]{3}{*}{$\mathrm{PAH}$} & \multicolumn{4}{|c|}{ maximum mineralization rate $\left(\%{ }^{14} \mathrm{C}\right.$ per day $)$} & \multicolumn{6}{|c|}{ PAH concentration $\left(\mathrm{mg} \mathrm{kg}^{-1}\right)$} \\
\hline & \multicolumn{2}{|c|}{ biostimulated $^{a}$} & \multicolumn{2}{|c|}{ inoculated $^{a}$} & \multirow[t]{2}{*}{ initial $^{b}$} & \multirow[t]{2}{*}{ predicted $^{c}$} & \multicolumn{2}{|c|}{ biostimulated $^{a, d}$} & \multicolumn{2}{|c|}{ inoculated $^{a, d}$} \\
\hline & control & + Brij 35 & control & + Brij 35 & & & control & + Brij 35 & control & + Brij 35 \\
\hline phenanthrene & $12.8 \pm 2.4$ & $21.1 \pm 0.7$ & $9.1 \pm 0.0$ & $18.9 \pm 0.6$ & $1329.5 \pm 52.7$ & 51.8 & $9.0 \pm 1.8$ & $<\mathrm{LD}^{e}$ & $19.3 \pm 3.5$ & $<\mathrm{LD}^{e}$ \\
\hline anthracene & $6.8 \pm 0.6$ & $9.1 \pm 6.1$ & $\mathrm{ND}^{f}$ & $\mathrm{ND}^{f}$ & $286.3 \pm 9.1$ & 15.4 & $22.4 \pm 1.1$ & $3.7^{g}$ & $89.0 \pm 30.1$ & $21.9 \pm 3.1$ \\
\hline fluoranthene & $5.1 \pm 0.4$ & $8.3 \pm 0.7$ & $28.5 \pm 2.6$ & $34.2 \pm 6.4$ & $881.5 \pm 3.4$ & 38.7 & $44.6 \pm 3.1$ & $20.0 \pm 3.4$ & $114.7 \pm 30.7$ & $7.8 \pm 0.1$ \\
\hline pyrene & $5.3 \pm 1.4$ & $9.1 \pm 1.2$ & $10.3 \pm 0.0$ & $23.0 \pm 0.8$ & $395.2 \pm 15.0$ & 30.4 & $17.9 \pm 0.0$ & $24.7 \pm 4.8$ & $88.6 \pm 1.4$ & $<\mathrm{LD}^{e}$ \\
\hline benzo[a]pyrene & $\mathrm{ND}^{f}$ & $\mathrm{ND}^{f}$ & $\mathrm{ND}^{f}$ & $\mathrm{ND}^{f}$ & $30.7 \pm 4.4$ & 4.1 & $16.6 \pm 1.7$ & $28.0 \pm 1.1$ & $20.9 \pm 1.3$ & $21.5 \pm 0.5$ \\
\hline$\Sigma \mathrm{PAH}^{h}$ & $\mathrm{ND}^{f}$ & $\mathrm{ND}^{f}$ & $\mathrm{ND}^{f}$ & $\mathrm{ND}^{f}$ & 2923.2 & 140.4 & 110.5 & 76.4 & 332.5 & 51.2 \\
\hline
\end{tabular}

${ }^{a}$ Biostimulated and inoculated denote, respectively, whether the microbial population responsible for the transformation was composed of autochthonous microorganisms or inoculated Mycobacterium gilvum VM552 cells. ${ }^{b}$ Initial PAH concentrations from Table 1 are also included here for a better comparison. ${ }^{c}$ Predicted concentration assuming that biodegradation acted only upon fast-desorption PAHs. Calculated with average $F_{\text {fast }}$ values from fittings in Table $1 .{ }^{d}$ Mass balance of the native compound remaining in the soil and in the aqueous phase after treatment. ${ }^{e}<\mathrm{LD}$, below limit of detection (in $\mathrm{mg} \mathrm{kg}^{-1}: 2.1$ for phenanthrene, 0.5 for pyrene). ${ }^{f} \mathrm{ND}$, not determined. ${ }^{g}$ Compound detected in only one duplicate. Limit of detection for anthracene: $1.0 \mathrm{mg} \mathrm{kg}^{-1} \cdot{ }^{h}$ Sum of five PAH: phenanthrene, anthracene, fluoranthene, pyrene and benzo[a]pyrene.

Table 3. Effect of an Anionic Surfactant (Brij 35, $20 \mathrm{~g} \mathrm{~L}^{-1}$ ) on Biodegradation of Polycyclic Aromatic Hydrocarbons (PAHs) in Suspensions of a Bioremediated Soil from a Manufactured Gas Plant Site (Soilrem)

\begin{tabular}{|c|c|c|c|c|c|c|}
\hline \multirow[b]{2}{*}{$\mathrm{PAH}$} & \multicolumn{2}{|c|}{ maximum mineralization rate $\left(\%{ }^{14} \mathrm{C}\right.$ per day) } & \multicolumn{4}{|c|}{ PAH concentration $\left(\mathrm{mg} \mathrm{kg}^{-1}\right)$} \\
\hline & control & + Brij 35 & initial $^{a}$ & predicted $^{b}$ & control $^{c}$ & + Brij $35^{c}$ \\
\hline phenanthrene & $6.7 \pm 1.3$ & $6.5 \pm 0.0$ & $42.3 \pm 5.6$ & 37.2 & $43.5 \pm 1.2$ & $18.2 \pm 0.6$ \\
\hline anthracene & $3.2 \pm 1.8$ & $4.1 \pm 0.1$ & $15.4 \pm 1.5$ & 13.9 & $15.5 \pm 1.5$ & $7.8 \pm 1.4$ \\
\hline fluoranthene & $4.4 \pm 0.5$ & $2.6 \pm 0.4$ & $54.8 \pm 4.3$ & 44.8 & $48.1 \pm 0.5$ & $26.0 \pm 3.7$ \\
\hline pyrene & $6.8 \pm 0.0$ & $3.6 \pm 1.3$ & $48.3 \pm 6.7$ & 41.0 & $37.8 \pm 2.7$ & $7.6 \pm 2.0$ \\
\hline benzo[a]pyrene & $\mathrm{ND}^{d}$ & $\mathrm{ND}^{d}$ & $22.4 \pm 0.1$ & 19.3 & $22.0 \pm 0.4$ & $9.7 \pm 1.5$ \\
\hline$\Sigma \mathrm{PAH}^{e}$ & $\mathrm{ND}^{d}$ & $\mathrm{ND}^{d}$ & 183.2 & 156.2 & 166.9 & 69.3 \\
\hline
\end{tabular}

${ }^{a}$ Initial PAH concentrations from Table 1 are also included here for a better comparison. ${ }^{b}$ Predicted concentration assuming that biodegradation acted only upon fast-desorption PAHs. Calculated with average $F_{\text {fast }}$ values from fittings in Table $1 .{ }^{c}$ Mass balance of the native compound remaining in the soil and in the aqueous phase after treatment. ${ }^{d} \mathrm{ND}$, not determined. ${ }^{e}$ Sum of five PAH: phenanthrene, anthracene, fluoranthene, pyrene, and benzo[a]pyrene.

of mineralization of the four PAHs, and a slight decrease in the maximum rates of mineralization of ${ }^{14} \mathrm{C}$-fluoranthene and ${ }^{14} \mathrm{C}$ pyrene (Table 3 ). Only $10 \%$ of the remaining benzo[a]pyrene was detected in the aqueous solutions from treatments with Brij 35 , possibly due to the active biodegradation of this $\mathrm{PAH}$ observed under these conditions. The surface tension of the aqueous phase yielded a value $\left(61.3 \pm 0.2 \mathrm{dyn}^{-1}\right)$ close to that observed with biostimulated, creosote-polluted soil, what also suggests biodegradation of the surfactant by the autochthonous microorganisms from MGP soil. Due to the observed effects of the surfactant on biodegradation of the slowly desorbing PAHs, the study with inoculated suspensions was considered not necessary with this soil.

\section{DISCUSSION}

The presence of slowly desorbing PAH fractions, as revealed by Tenax extraction, of the same order of magnitude as the residual $\mathrm{PAH}$ concentrations remaining after bioremediation indicates that microorganisms cannot overcome the bioavailability restrictions imposed by slow desorption. These observations agree with previous studies assessing the bioavailability of slowly desorbing PAHs to microbial populations able to degrade these chemicals. ${ }^{1,3}$ Our results extend those findings by indicating that a nontoxic, nonionic surfactant (Brij 35) can be used to enhance the biodegradation of slowly desorbing PAHs and, therefore, improve bioremediation performance. Measurements of solubilization of PAHs in the presence of surfactant solutions at concentrations above CMC yielded recoveries that evidenced the capacity of the surfactant to act on a significant fraction of slowly desorbing PAHs present in field-contaminated soils (Table 1). Biodegradation experiments also showed that this solubilization led to decreases in total PAH contents beyond $F_{\text {fast }}$ predictions (Tables 2 and 3 ). These results suggest that the surfactant may have efficiently acted upon compartments responsible in these field-contaminated soils for slow desorption, eventually semisolid, NAPL-like creosote materials, ${ }^{5}$ black carbon, ${ }^{6}$ or aged residues, ${ }^{7}$ possibly through interactions with its hydrophobic moiety, to increase biodegradation. Indeed, hydrophobic interactions between the surfactant Brij 30 and these field-contaminated soil constituents have been identified as the possible cause for the enhanced desorption and biodegradation of PAHs observed, at sub-CMC levels, in a previously bioremediated MGP soil. ${ }^{14}$

The surfactant caused an enhancement in the maximum rate of mineralization of PAHs in creosote-polluted soil (Figure 2 and Table 2). Given the prevalence of fast-desorbing PAHs in this soil, these results indicate that the surfactant was also effective in 
promoting biodegradation of this fraction. Because our focus was on slow desorption, we did not attempt a detailed study for the causes of this enhancement, what will be the subject for further research. However, the surfactant did not cause an enhancement of mineralization of PAHs in MGP soil, in spite of the significant decrease in PAH residual contents (Table 3). These results can be explained considering that, in creosote-polluted soil and in MGP soil incubated with surfactant, the ${ }^{14} \mathrm{C}$-labeled compounds were homogenized with the bioaccessible fraction of the native chemicals, and that ${ }^{14} \mathrm{CO}_{2}$ production not only corresponded to respiration of added ${ }^{14} \mathrm{C}$-PAH but also represented the biodegradation of bioaccessible ${ }^{12} \mathrm{C}$-PAHs. It is also possible that, due to a limited bioaccessibility of the native PAHs in MGP soil, only the added ${ }^{14} \mathrm{C}$-PAH were mineralized significantly in the surfactant-free controls, thus resulting in apparently higher mineralization rates (measured as \% of radiochemical added) than in the treatment with surfactant. These results confirmed the advantages of using radiorespirometry determinations coupled to the analysis of residual concentrations of native PAHs as a tool to quantify bioaccessible PAH fractions. Furthermore, they also evidenced the enhancing effect of the surfactant on the bioaccessibility of the highly recalcitrant PAHs present in bioremediated MGP soil.

Current knowledge on biodegradation of PAHs indicates that, of the 16 PAHs listed by the U.S. Environmental Protection Agency as priority pollutants, those with molecular weight up to 202_including the high-molecular-weight (HMW) PAHs pyrene and fluoranthene-can be degraded through growth-linked aerobic reactions - the rest (e.g., benzo[a]pyrene) being susceptible only to cometabolic attack. ${ }^{19}$ Besides, the biodegradability often observed in aerobic environments, such as many soils, shows a higher recalcitrance of PAHs composed of a progressive higher number of benzene rings. ${ }^{18}$ The enhancement of biodegradation of these highly recalcitrant HMW PAHs is therefore of considerable interest. Our results with pyrene and benzo[a]pyrene in biostimulated suspensions of creosote-polluted and MGP soils showed, however, that Brij 35 can influence the biodegradation of HMW PAHs in different ways. In creosotepolluted soil, the surfactant depressed the biodegradation, but it enhanced biodegradation when the chemicals were present in MGP soil. The dissimilar outcomes may be a consequence of the balance of two effects, namely an increase in bioaccessibility of the chemicals on the one hand and the enhancement of the rate of simultaneous consumption of the other PAHs, with subsequent competition effects, on the other. The latter effect is in agreement with findings showing that the rate of benzo[a]pyrene cometabolism by bacteria is negatively influenced by the simultaneous occurrence of growth-supporting PAHs, like anthracene and pyrene, which compete for a common enzymatic pathway, ${ }^{20}$ and with competition effects also observed during simultaneous biodegradation of growth-supporting PAHs. ${ }^{5}$ Alternately, partitioning of these HMW PAHs into surfactant micelles may have prevented biodegradation in the creosote-polluted soil due to limitations for uptake by microbial cells. However, surface tension measurements performed at the end of the assays evidenced, for biostimulated soils, that the surfactant was present in the aqueous phase as monomers, what suggests that this alternative explanation is unlikely, at least at these later stages of the experimental period.

The data suggest that the application of biodegradable surfactants as a way to improve the performance of bioremediation of PAHs may be more effective with contaminated soils that have been previously undergone extensive bioremediation to enrich for a slowly desorbing profile. The dissimilar relative concentrations of desorbed benzo[a]pyrene detected in biostimulated, surfactant-containing slurries of both soils suggest that this strategy would not only avoid the inhibition of biodegradation of HMW PAHs through the competition mechanisms described above; It also would allow the minimization of risks associated to an increased chemical activity and toxicity of remaining HMW PAHs, caused by surfactant solubilization. Other advantages of this late application may relate to the biodegradable nature of the surfactant, what is obviously necessary to minimize environmental impacts. They include eventual increases in nutrient and oxygen demands, what would be less important at lower pollutant levels, and, as evidenced by surface tension measurements in this study, the loss of surfactant efficiency at later stages of treatment-when it is really needed to act upon slowly desorbing PAHs. The assessment of the kinetics of PAH desorption, to determine the magnitude of the slowly desorbing fractions present in the polluted soil, seems to be a prerequisite for this optimized surfactant role in bioremediation. In addition, optimization should obviously consider other aspects, such as applicability to a wider number of remediation situations, surfactant performance in solid-phase systems, and costs and environmental impact of the surfactant.

\section{ASSOCIATED CONTENT}

S Supporting Information. Kinetics of desorption; Effect of sorption of the surfactant Brij 35 onto creosote-polluted soil on surface tension of aqueous solutions; Effect of surfactant on biodegradation of PAHs in MGP soil. This material is available free of charge via the Internet at http://pubs.acs.org.

\section{AUTHOR INFORMATION}

\section{Corresponding Author}

*Phone: (+34) 95-4624711; fax: (+34) 95-4624002; e-mail: jjortega@irnase.csic.es.

\section{ACKNOWLEDGMENT}

We thank EMGRISA and Soilrem for the provision of the soil samples. Support for this research was provided by Spanish Ministry of Science and Innovation (CGL2007-64199/BOS), Junta de Andalucía (PAI RNM 312) and European Union (contract QLRT-1999-00326).

\section{REFERENCES}

(1) Cornelissen, G.; Rigterink, H.; Ferdinandy, M. M. A.; Van Noort, P. C. M. Rapidly desorbing fractions of PAHs in contaminated sediments as a predictor of the extent of bioremediation. Environ. Sci. Technol. 1998, 32, 966-970.

(2) Reichenberg, F.; Karlson, U. G.; Gustafsson, O.; Long, S. M.; Pritchard, P. H.; Mayer, P. Low accessibility and chemical activity of $\mathrm{PAHs}$ restrict bioremediation and risk of exposure in a manufactured gas plant soil. Environ. Pollut. 2010, 158, 1214-1220.

(3) Gomez-Lahoz, C.; Ortega-Calvo, J. J. Effect of slow desorption on the kinetics of biodegradation of polycyclic aromatic hydrocarbons. Environ. Sci. Technol. 2005, 39, 8776-8783.

(4) Ortega-Calvo, J. J.; Alexander, M. Roles of bacterial attachment and spontaneous partitioning in the biodegradation of naphthalene initially present in nonaqueous-phase liquids. Appl. Environ. Microbiol. 1994, 60, 2643-2646. 
(5) Lopez, Z.; Vila, J.; Ortega-Calvo, J. J.; Grifoll, M. Simultaneous biodegradation of creosote-polycyclic aromatic hydrocarbons by a pyrene-degrading Mycobacterium. Appl. Microbiol. Biotechnol. 2008, $78,165-172$.

(6) Ortega-Calvo, J.-J.; Gschwend, P. M. Influence of low oxygen tensions and sorption to sediment black carbon on biodegradation of pyrene. Appl. Environ. Microbiol. 2010, 76, 4430-4437.

(7) Hatzinger, P. B.; Alexander, M. Effect of aging of chemicals in soil on their biodegradability and extractability. Environ. Sci. Technol. 1995, $29,537-545$.

(8) Garcia, J. M.; Wick, L. Y.; Harms, H. Influence of the nonionic surfactant Brij 35 on the bioavailability of solid and sorbed Dibenzofuran Environ. Sci. Technol. 2001, 35, 2033-2039.

(9) Kim, I.; Park, J. S.; Kim, K. W. Enhanced biodegradation of polycyclic aromatic hydrocarbons using nonionic surfactants in soil slurry. Appl. Geochem. 2001, 16, 1419-1428.

(10) Liu, Z.; Edwards, D. A.; Luthy, R. G. Sorption of non-ionic surfactants onto soil. Water Res. 1992, 26, 1337-1345.

(11) Liu, Z.; Jacobson, A. M.; Luthy, R. G. Biodegradation of naphthalene in aqueous nonionic surfactant systems. Appl. Environ. Microbiol. 1995, 61, 145-151.

(12) Liu, Z.; Laha, S.; Luthy, R. G. Surfactant solubilization of polycyclic aromatic hydrocarbon compounds in soil-water suspensions. Water Sci. Technol. 1991, 23, 475-485.

(13) Yeom, I. T.; Ghosh, M. M.; Cox, C. D. Kinetics aspects of surfactant solubilization of soil-bound polycyclic aromatic hydrocarbons. Environ. Sci. Technol. 1996, 30, 1589-1595.

(14) Zhu, H.; Aitken, M. D. Surfactant-enhanced desorption and biodegradation of polycyclic aromatic hydrocarbons in contaminated soil. Environ. Sci. Technol. 2010, 44, 7260-7265.

(15) Niqui-Arroyo, J. L.; Ortega-Calvo, J. J. Integrating biodegradation and electroosmosis for the enhanced removal of polycyclic aromatic hydrocarbons from creosote-polluted soils. J. Environ. Qual. 2007, $36,1444-1451$.

(16) Niqui-Arroyo, J. L.; Ortega-Calvo, J. J. Effect of electrokinetics on the bioaccessibility of polycyclic aromatic hydrocarbons in polluted soils. J. Environ. Qual. 2010, 39, 1993-1998.

(17) Posada-Baquero, R.; Niqui-Arroyo, J. L.; Bueno-Montes, M.; Gutierrez-Daban, A.; Ortega-Calvo, J. J. Dual C-14/residue analysis method to assess the microbial accessibility of native phenanthrene in environmental samples. Environ. Geochem. Health 2008, 30, 159-163.

(18) Uyttebroek, M.; Spoden, A.; Ortega-Calvo, J. J.; Wouters, K.; Wattiau, P.; Bastiaens, L.; Springael, D. Differential responses of eubacterial, Mycobacterium, and Sphingomonas communities in polycyclic aromatic hydrocarbon (PAH)-contaminated soil to artificially induced changes in PAH profile. J. Environ. Qual. 2007, 36, 1403-1411.

(19) Kanaly, R. A.; Harayama, S. Biodegradation of high-molecularweight polycyclic aromatic hydrocarbons by bacteria. J. Bacteriol. 2000, 182, 2059-2067.

(20) McLellan, S. L.; Warshawsky, D.; Shann, J. R. The effect of polycyclic aromatic hydrocarbons on the degradation of benzo[a]pyrene by Mycobacterium sp. strain RJGII-135. Environ. Toxicol. Chem. 2002, 21, 253-259. 